\title{
Xenogeneic Humoral Graft-Vs-Host Disease Following Hamster-to-Rat Bone Marrow Transplantation
}

\author{
T. Miki, Y.-H. Lee, A. Tandin, V. Subbotin, R. Kuddus, A.S. Rao, J.J. Fung, T.E. Starzl, and L.A. Valdivia
}

$\mathrm{B}_{\text {essi }}$ ONE marrow transplantation (BMT) is an effective strategy to induce tolerance across xenogeneic barriBMT model. ${ }^{2}$ However, all animals suffered from graft-vshost disease (GVHD). Although xenogeneic GVHD has been observed in other studies, it is not yet well characterized. ${ }^{3}$ Because xenograft rejection is primarily mediated by humoral mechanisms, we tested the hypothesis that, unlike allogeneic GVHD in which cellular mechanisms predominate, xenogeneic GVHD could also be humorally mediated.

\section{MATERIALS AND METHODS}

LEW rats underwent splenectomy (Spx) on day -7 and total body irradiation (TBI) of 10 Gy 3 days before BMT. $300 \times 10^{6}$ unfractionated hamster bone marrow cells were infused intravenously (IV). Rat recipients were divided into three groups: shortterm ( 7 days) and long-term ( 30 days) treatment groups (groups I and II, respectively) received tacrolimus $1 \mathrm{mg} / \mathrm{kg} / \mathrm{d}$ intramuscularly beginning 1 day before BMT, whereas the control group (group III) did not receive any immunosuppressive agents. All recipients were observed for clinical signs of GVHD. Chimerism was detected by two-color flow cytometry using an FITC-conjugated rat antihamster lymphocyte antibody and a biotinylated hamster anti-rat lymphocyte antibody. Cytotoxicity of recipient's sera against rat and hamster cells was measured by complement-dependent cytotoxicity (CDC).

Several samples were treated with dithiothreitol (DTT) to determine whether IgM or IgG was responsible for the cytotoxicity. Histopathologic studies were done with $\mathrm{H} \& \mathrm{E}$. Immunofluorescence staining was performed to detect hamster anti-rat Ig on several organs.

\section{RESULTS}

Hamster bone marrow cells engrafted only in $25 \%$ of rats in the control group; more than $90 \%$ did so in both short-term and long-term tacrolimus treatment groups. In group I, six of nine animals showed dermatitis and three rats died around day 28 post-BMT as a result of humoral injury to the liver. In group II, none of the animals developed dermatitis, but they all developed fatal humoral liver injury. Overall survival for animals in group II was shorter than that in group I (a median of 30.3 vs 53.7, respectively; $P=.073$ ).

Flow cytometric analysis of peripheral blood lymphocytes showed more than $80 \%$ in both group I $(86.5 \pm 2.5 \%)$ and group II $(88.7 \pm 3 \%)$ at 4 weeks post-BMT. Humoral injury was suspected because some rat liver samples had extensive infarction with focal hepatocyte necrosis in zones 1 and 2 , in addition to infiltration by polymorphonuclear leukocytes. The central vein and pericentral hepatocytes (zone 3 ) were not compromised. There were no signs of obvious humoral injury in other organs. Immunofluorescence staining showed hamster anti-rat immunogloblin deposition on the rat portal vein and focal necrotic area. In no other organs was such extensive deposition of antibody found. CDC test using sera from naive rats and hamsters showed that xenoreactive natural antibodies (XNA) were present in both species against each other. Anti-hamster XNA disappeared in BMT recipients. However, cytotoxic antibody titers against rat lymphocytes were found in some of the rats suffering from humoral injury to the liver, which were sensitive to DTT treatment.

\section{DISCUSSION}

This is the first evidence of humoral GVHD in xenogeneic BMT. There are several reports of successful mixed lymphohematopoietic chimerism in rat-to-mouse ${ }^{1}$ and pig-tomouse animal combinations, but there is no description of humoral GVHD. Mice are not as prone to mount a deleterious humoral response due to deficiencies in several proteins of the complement system. ${ }^{4}$ Our data suggest that humoral GVHD can occur in other species where complement is more efficient, namely, the rat.

The addition of tacrolimus treatment markedly increased the engraftment rate. However, the outcome differed with different lengths of tacrolimus administration. Rats receiving a 1-week course of tacrolimus developed cellular GVHD, expressed with symptoms similar to those of allogeneic GVHD in the rat. ${ }^{5}$ These symptoms were absent in rats that received a long-term course of tacrolimus treatment. Interestingly, these rats had severe liver damage that

From the Thomas E. StarzI Transplantation Institute, University of Pittsburgh Medical Center, Pittsburgh, Pennsylvania.

Address reprint requests to Luis $A$. Valdivia, MD, PhD, TESTI, E-1546 Biomedical Science Tower, 200 Lothrop St, Pittsburgh, PA 15261. 
was humorally mediated. DTT assay data suggest that this humoral injury was mediated by hamster IgM.

We conclude that xenogeneic GVHD in our model may have a dual presentation: classical cellular GVHD not distinct to the allogeneic BMT and humoral GVHD. Although cellular GVHD is a generalized phenomenon, the liver is the target organ in humoral GVHD and the degree of injury is potentiated by $\mathrm{T}$-cell suppression. These findings suggest that problems regarding humoral GVHD must be considered in xenogeneic BMT.

\section{REFERENCES}

1. Ildstad ST, Wren SM, Boggs SS, et al: J Exp Med 174:467, 1991

2. Patijn GA, Valdivia LA, Pan F, et al: Transplantation 57: 1528,1994

3. Bouwman E, Scheringa M, Marquet RL: Transplant Proc 28:681, 1996

4. Baldwin WM 3rd, Pruitt SK, Brauer RB, et al: Transplantation 59:797, 1995

5. Markus PM, Cai X, Ming W, et al: Transplantation 52:590, 1991 